Dickenson, P. B. \& Macdonald, K. D. (1955). J. gen. Microbiol. 13, 84-90.

\title{
An Electron Microscope Examination of the Initial Cell Stage in Streptomyces spp.
}

\author{
By P. B. DICKENSON and K. D. MACDONALD \\ Glaxo Laboratories Ltd., Fermentation Research Division, \\ Sefton Park, Stoke Poges, Buckinghamshire
}

SUMMARY : Electron microscope observations were made on submerged cultures in defined medium of two species of the genus Streptomyces. The evidence obtained confirms the supposition of Klieneberger-Nobel (1947), with surface cultures of four other species, that at an early stage. in the life history of the organism concerned, fusion occurs between portions of the same or different hyphae, culminating in the formation of 'initial cells'. In S. griseus initial cells were observed at $26 \mathrm{hr}$. and as late as 6 days. Certain unidentified bodies were observed. These may be related to initial cell formation.

It has been known for some time that species of the genus Streptomyces show two different growth phases-the substratum mycelium and the aerial mycelium (Ørskov, 1923). These phases were renamed the primary and the secondary mycelium, respectively, by Kleineberger-Nobel (1947); her terminology will be used in this paper. The recognition of diphasic growth, along with a persistent branching filamentous habit and reproduction from spores, suggests, according to Bisset \& Moore (1949), a clear separation from the other Actinomycetales (Bergey's Manual), and on this basis the 'four sporing Actinomycetes' described by Klieneberger-Nobel in her paper may be considered as species of the genus Streptomyces. This author made the first critical examination of the life cycle of these organisms as it occurs in surface cultures on a solid agar medium or on a liquid medium. By combining a staining method based upon that of Robinow (1942) with careful microscopical examination, she found a marked difference in the two phases of growth in the species examined. The primary mycelium, developed from germinating spores, is septate and profusely branching, and the individual hyphae, which are somewhat less than $1 \mu$. in diameter, contain 'granular or rod-shaped chromatin bodies'. The secondary spore-bearing mycelium, as she describes it, arises directly from the primary mycelium in agglomerations of the latter and from points of fusion which Klieneberger-Nobel terms 'initial cells'; it is distinguished in that it is thicker, branches less profusely, eventually breaks into single cells or spores and has a more clearly defined internal structure. According to Klieneberger-Nobel the spores are developed after fusion and organization of the cellular material, processes whose exact significance is not yet apparent. Klieneberger-Nobel suggested that the complete cycle might represent alternating generations of haploid primary mycelium with diploid secondary mycelium, with sexual fusion occurring in the initial cell, but stated that "in order to prove this an analysis of "chromosome" 
content complemented by observations proving the actual fusion of chromatin would be required. Such evidence will be difficult to obtain, since all the elements concerned are of exceedingly small dimensions'.

Similar observations were made on the closely related genus Micromonospora by Morris (1952); and more recently McGregor (1954), in describing certain aspects of the life cycle of an unspecified Streptomyces strain, generally agreed with Klieneberger-Nobel's findings, but differed in that he considered that initial cells are not formed from 'nests of filaments' and appeared to arise, not from points of hyphal fusion, but from single swellings in the hyphae or as short appendages. Klieneberger-Nobel's conclusions were strongly disputed by Erikson (1949), on the grounds that hyphal agglomerations are artefacts inevitably caused in the preparation of the microscopical specimens, further, she stated that in her experience, 'in no circumstance has any fusion been detected between filaments of the same or different colonies'. 'The secondary mycelium, in Erikson's view, arises directly from the primary mycelium, without the intervention of an initial cell stage. However, Erikson did mention, without comment, that Carvajal (1946) observed hyphal fusion during electron microscope studies of Streptomyces griseus, though this author provided no photographic or descriptive evidence for this statement. It is clear that much of the doubt as to the nature and function of the 'initial cell' lies in the difficulty of interpreting the appearance of objects close in size to the limit of resolution of an ordinary microscope. The purpose of the present paper, which describes electron microscopic observations on streptomycin- and vitamin $B_{12}$-producing strains of $S$. griseus and of a strain of $S$. aureofaciens, is to present alternative evidence for the existence of these structures. In addition, we have recorded certain other observations on the morphology of Streptomyces spp.

\section{METHODS}

Most of our observations were made on material grown in agitated submerged culture in which, with certain media (Carvajal, 1947), sporulation readily occurs. The cycle of events leading to sporulation is then fundamentally the same as that observed for agar surface cultures.

Cultures were grown in shaken $250 \mathrm{ml}$. conical flasks containing $40 \mathrm{ml}$. medium, of which two kinds were used. The first (urea medium) consisted of glucose, 5 g.; brown sugar, 10 g.; urea, 2 g.; $\mathrm{KH}_{2} \mathrm{PO}_{4}, 2 \mathrm{~g}$.; with water to $1000 \mathrm{ml}$. and had a $\mathrm{pH}$ value of $6 \cdot 8$ (after sterilization at $120^{\circ}$ for $20 \mathrm{~min}$.). The second (AS medium) consisted of glucose, $25 \mathrm{~g}$.; $\mathrm{MgSO}_{4} \cdot \mathbf{7} \mathrm{H}_{2} \mathrm{O}, \mathbf{1} \mathrm{g}$.; $\left(\mathrm{NH}_{4}\right)_{2} \mathrm{SO}_{4}, 4 \mathrm{~g}$; $\mathrm{NaNO}_{3}, 5 \mathrm{~g}$; $\mathrm{NaCl}, 5 \mathrm{~g}$; $\mathrm{KH}_{2} \mathrm{PO}_{4}, 0.5 \mathrm{~g}$; $\mathrm{K}_{2} \mathrm{HPO}_{4}, 1 \mathrm{~g}$; $\mathrm{CaCO}_{3}, 0 \cdot 45 \mathrm{~g}$; trace elements in the form of soluble salts of iron, manganese, zinc, copper and molybdenum at the rate of 3 p.p.m. of each element; with water to $1000 \mathrm{ml}$. This had a $\mathrm{pH}$ value of 7 (after sterilization at $120^{\circ}$ for 20 min.). Flasks were inoculated with spores from surface cultures of the organism concerned and grown at $28^{\circ}$ on a rotary shaker (200 r.p.m.).

Electron microscope examinations of Streptomyces griseus and $S$. aureofaciens were made exclusively on formalin-fixed material. Material for visual 
observations was fixed in osmium tetroxide vapour and stained by the $\mathrm{HCl}$ Giemsa method of Robinow (1942). Random electron microscope observations were made directly, without metal shadow-casting, on samples of $S$. griseus ranging from $26 \mathrm{hr}$. to 6 days in age, and of $S$. aureofaciens 46 and $53 \mathrm{hr}$. old. All samples were mounted, air-dried, on formvar films after washing six times by centrifugation in distilled water, a procedure found to be necessary to eliminate traces of dissolved medium constituents which interfered with the clarity of the electron micrograph. Most of the observations were made at an accelerating voltage of $60 \mathrm{kV}$., though some were made at $80 \mathrm{kV}$. The resulting prints vary in magnification between 2500 diameters and 12,000 diameters, but that recorded is to some extent dependent on the accelerating voltage, which, though stable over an operating period, may vary slightly from day to day, so that the figures are not exact, but near enough for comparative purposes. The ordinary light photomicrographs are reproduced at a magnification of 2000 diameters. The electron microscope used was the Philips instrument in the Department of Biophysics at Leeds University, and the ordinary photomicrographs were made with a Zeiss 1.3 N.A., $\times 100$ fluorite oil-immersion lens, a Leitz $\times 10$ Periplan eyepiece, a 1.4 N.A. Zeiss aplanatic condenser and a Leitz Makam camera attachment, with Köhler illumination and green or blue Ilford filters.

\section{RESULTS}

With visual light examination, the appearance of the initial cell stage is illustrated in Pl. 1, fig. 1, which is of Streptomyces griseus primary mycelium aged $48 \mathrm{hr}$. Four main features are observable, and are indicated on the photomicrograph by the lettered annotations: $(a)$ initial cells may arise at the point of fusion of separate hyphae; $(b)$ they may arise in an intercalary position on individual hyphae without fusion with other hyphae; $(c)$ they develop as short appendages to individual hyphae; $(d)$ they may break away free from the mycelium. In whatever position they arise, initial cells appear to fulfil the same function of initiating the secondary mycelium, as illustrated in Pl. 1, fig. 2, where the first stage in 'germination' of an initial cell is clearly seen. With a gradual elongation and thickening of the 'germ-tube' the hypha then assumes the characteristic appearance of secondary mycelium (Pl. 1, fig. 3; see also Klieneberger-Nobel, 1947, pl. 2, fig. 13). In the species described by Klieneberger-Nobel, hyphal fusion leading to initial cell formation seems to occur in a tangled 'nest of filaments', but we observed this feature only occasionally (Pl. 1, fig. 4). The functional significance of this is not obvious; it may be a fortuitous agglomeration of free initial cells.

When viewed directly with an electron microscope after formalin-fixation, the mycelium varies in its opacity to the electron beam; internal structure is only occasionally visible, but useful information may be obtained from 'shadow' impressions. Generally the internal structure revealed has the appearance shown in Pl. 2, figs. 7, 8. Material dense to transmitted light is not necessarily dense to electrons, but the texture here is reminiscent of vacuolated cytoplasm as observed by conventional methods. The number and diversity in size of the 
less dense patches suggest that they are vacuoles and preclude their being nuclei. The various features observed will be dealt with under subheadings; as they have commonly been observed in specimens of either species, this will not be specified in the text, but may be found from the legends of the figures.

Hyphal fusion. Evidence for the occurrence of hyphal fusion is strengthened, as will be seen by reference to the portions indicated by arrows in Pl. 1, fig. 5, and Pl. 2, fig. 6. It might be argued that the hyphae simply assumed close parallel positions in the preparation of the specimen, and this undoubtedly does occur through surface tension effects during drying on the electron microscope mount. However, in direct observation on the screen it was possible to differentiate between this appearance and that of Pl. 1, fig. 5, and Pl. 2, fig. 6, where the bridge between the hyphae consists of a short outgrowth from one lying close to the other, presumably just before fusion takes place along the contact boundary. Reference to Pl. $\mathbf{1}$, fig. 5, will show that there is an unmistakable thickening in the adjacent (arrowed) portion, which may indicate a positive reaction caused in one hypha by the proximity of another.

Initial cells. It seems that hyphal fusions between different hyphae, as described above, or between different portions of the same hypha, in which the eventual coalescence of material has varying degrees of complexity, are often the first stages in the formation of initial cells: Pl. 2, figs. 7-9, and Pl. 3, fig. 10, illustrate this thesis. The bodies illustrated represent the first stages of initial cell formation from double (Pl. 2, fig. 7), treble (Pl. 2, fig. 8) and multiple (Pl. 2, fig. 9) hyphal contacts, and Pl. 3, fig. 10 represents a late stage of an initial cell limited to one hypha (see also Pl. 1, fig. $1 b$ ).

Pl. 2, figs. 7, 8, confirm the opinion formed from visual light observations (Pl. 1, fig. I $d$, and McGregor, 1954) that initial cells may become detached from the main mass of mycelium. This seems to take place at thinned portions of the hyphae, which occur at irregular intervals along their length (Pl. 2, fig. 9 ; Pl. 3, figs. 11, 12), thus accounting for the tapered extremities in Pl. 2, figs. 7,8 .

In our cultures the presence of initial cells is not limited to the early stages of the fermentation, although it is limited to the early stages of the life cycle. This is borne out by their presence as late as 6 days (Pl. 2, figs. 7, 8) after inoculation of cultures of Streptomyces griseus, and indeed this is not surprising, since Carvajal (1947) in his early study of submerged cultures found that spores formed under such conditions can germinate late in the same fermentation and regenerate fresh mycelium. Clearly, in our material it is this fresh primary mycelium that has given rise to the late-formed initial cells.

Unidentified objects. Several bodies seen could not immediately be related to any views based upon visual light examinations. One of these is shown in Pl. 3, fig. 13 (arrowed): it is ovoid in shape, transparent to electrons and flanked by two thickened portions of the parent hypha. From each end arises a single short filament of the same thickness as the unthickened parent hypha, forming a crossed pair at one side of the ovoid. It may be that the whole apparatus represents the germination of a single initial cell in situ in its parent 
hypha, but the direction in which the two short filaments (possibly germ tubes) are growing is hardly what one would expect if this were so. An alternative possibility is that the structure may represent hyphal regeneration from a damaged portion.

In other examples (Pl. 3, figs. 11, 12) portions of hyphae opaque to electrons give way to sections that are electron transparent and of unknown significance.

\section{DISCUSSION}

The presence of a fusion initial cell stage in the life history of Streptomyces spp. prompts speculation as to its function, for it is suggestive of a haploid primary mycelial stage leading after nuclear fusion in the initial cell to a diploid secondary mycelial stage. Since it seems clear from the work described above that initial cells can arise either within a single hypha or from fused portions of two or more hyphae, then nuclear fusion, if it takes place, is likely to be an intra-mycelial as well as an inter-mycelial event.

An hypothesis of a haploid/diploid alternation was tentatively put forward by Klieneberger-Nobel (1947), who qualified it with the reservation, previously quoted, that the evidence would be difficult to obtain because of the exceedingly small size of the elements concerned. We agree with this view, and indeed extend it, for it is thought that visual microscope methods are unlikely to resolve the problem with certainty. Nor would electron microscope observation; by the nature of preparation by desiccation and the disruptive effect of the electron stream on cellular material, cytological observations by these methods are difficult to interpret. In view of this doubt, it would be preferable to investigate the problem by genetical rather than cytological methods. By such means, based upon the methods of Pontecorvo (1953) for the genetical analysis of homothallic fungi, it might be possible to establish the presence or otherwise of a true sexual fusion.

If there were a true alternation of generations, then at some point before spore formation, or soon after, there would be a restoration of the haploid state through the agency of a meiotic division. Indeed, there is some evidence that meiosis may not be a pre-sporulation event, but that it occurs at least after spore maturation. This is suggested by experimental work carried out by us on spore material from surface cultures of Streptomyces griseus in which attempts were made to produce nutritional mutant strains by the usual techniques (Pontecorvo, 1953). No nutritional mutants were retrieved from among 1600 ultraviolet irradiation survivors. Their complete absence could be ascribed to a variety of causes, of which the most likely are a high resistance to the mutagenic effects of ultraviolet light or spores that are diploid or multinucleate.

The first of these possibilities is thought to be unlikely, for here and elsewhere the mutagenic effect of ultraviolet light on Streptomyces griseus has been demonstrated for mutations involving a change in streptomycin or vitamin $\mathbf{B}_{12}$ productivity or pigment formation or morphological changes.

On the other hand, if the spores irradiated were diploid or multinucleate the 
possibility of retrieving nutritional mutants by the methods we employed would be small, since in a diploid cell, two mutations involving each allele of a particular locus would be necessary for the phenotypic expression of a particular growth factor requirement, whereas in a haploid binucleate cell, for example, two mutations at the same locus in each nucleus would be required. There is, however, cytological evidence that the spores of Streptomyces spp. are not multinucleate (Klieneberger-Nobel, 1947; McGregor, 1954; our observations).

We wish to express our thanks to Professor R. D. Preston of the Department of Biophysics, Leeds University, for the facilities he provided; to Mr G. W. Ripley and Mr R. W. McWilliam for technical assistance, and to Dr B. Northam and Dr A. Rhodes for the supply of material.

\section{REFERENCES}

Bergey's Manual of Determinative Bacteriology (1948). 6th edition. Edited by Breed, R. S., Murray, E. G. D. \& Hitchens, A. P. London: Baillière, Tindall and Cox.

Bisset, K. A. \& Moone, F. W. (1949). The relationship of certain branched bacterial genera. J. gen. Microbiol. 3, 387.

Carvajal, F. (1946). Studies on the structure of Streptomyces griseus. Mycologia, $38,587$.

Carvajal, F. (1947). The production of spores in submerged cultures by some Streptomyces. Mycologia, 39, 426.

Erikson, D. (1949). The morphology, cytology, and taxonomy of the Actinomycetes. Annu. Rev. Microbiol. 3, 23.

Khieneberger-Nobel, E. (1947). The life cycle of sporing Actinomyces as revealed by a study of their structure and septation. J. gen. Microbiol. 1, 22.

McGregor, J. F. (1954). Nuclear division and the life cycle in a Streptomyces sp. J. gen. Microbiol. 11, 52.

Mornis, E. O. (1952). The cytology of the filamentous bacteria. Chem. \& Ind. p. 120.

Ørskov, K. (1923). Investigations into the Morphology of the Ray Fungi. Copenhagen: Levin and Munksgaard.

Ponteconvo, G. (1953). The genetics of Aspergillus nidulans. Advanc. Genet. 5, 141.

Robinow, C. F. (1942). A study of the nuclear apparatus of bacteria. Proc. roy. Soc. B, 130, 299.

\section{EXPLANATION OF PLATES}

\section{Plate 1}

Fig. 1. S. griseus : primary mycelium at $48 \mathrm{hr}$. from submerged culture showing initial cells at $a, b, c, d$, and other positions. Osmium tetroxide fixation (HCl-Giesma staining). Visual light, $\times 2000$. Urea medium.

Fig. 2. S. griseus: primary mycelium at $40 \mathrm{hr}$. from submerged culture, showing germinating initial cell. Osmium tetroxide fixation (HCl-Giemsa staining). Visual light, $\times 2000$. A.S. medium.

Fig. 3. S. griseus: primary mycelium at $44 \mathrm{hr}$. from submerged culture showing elongation of secondary mycelium from an initial cell. Osmium-tetroxide fixation (HCl-Giemsa staining). Visual light, $\times 2000$. A.S. medium.

Fig. 4. S. griseus: primary mycelium at $40 \mathrm{hr}$. from submerged culture, showing agglomeration of hyphae. Osmium tetroxide fixation (HCl-Giemsa staining). Visual light, $\times 2000$. Urea medium.

Fig. 5. S. aureofaciens: primary mycelium at $53 \mathrm{hr}$. from submerged culture, showing hyphal fusions. Electron micrograph, $\times 6000$. Urea medium. 


\section{Plate 2}

Fig. 6. S. griseus: primary mycelium at $53 \mathrm{hr}$. from submerged culture, showing hyphal fusions. Electron micrograph, $\times 5000$. Urea medium.

Fig. 7. S. griseus: first stage of initial cell formation from double hyphal contact. From 6-day submerged culture. Electron micrograph, $\times 10,000$. (Inset: diagram illustrating conformation of hyphae.) Urea medium.

Fig. 8. S. griseus: first stage of initial cell formation from treble hyphal contact. From 6-day submerged culture. Electron micrograph, $\times 12,000$. (Inset: diagram illustrating conformation of hyphae.) Urea medium.

Fig. 9. S. griseus : early stage initial cell formation from multiple hyphal contacts, showing thinning portions of hyphae. From $26 \mathrm{hr}$. submerged culture. Electron micrograph, $\times 5000$. A.S. medium.

\section{Plate 3}

Fig. 10. S. aureofaciens: late stage initial cell formation within a single hypha. From $53 \mathrm{hr}$. submerged culture. Electron micrograph, $\times 10,000$. Urea medium.

Fig. 11. S. aureofaciens: thinned out portions of hyphae and electron transparent portions. From $46 \mathrm{hr}$. submerged culture. Electron micrograph, $\times 5000$. Urea medium.

Fig. 12. S. aureofaciens: thin portions of hyphae with electron transparent sections. From $26 \mathrm{hr}$. submerged culture. Electron micrograph, $\times 2500$. Urea medium.

Fig. 13. S. aureofaciens: electron transparent 'unidentified body'. From $53 \mathrm{hr}$. submerged culture. Electron micrograph, $\times 6000$. Urea medium. 
Journal of General Microbiology, Vol. 13, No. 1.
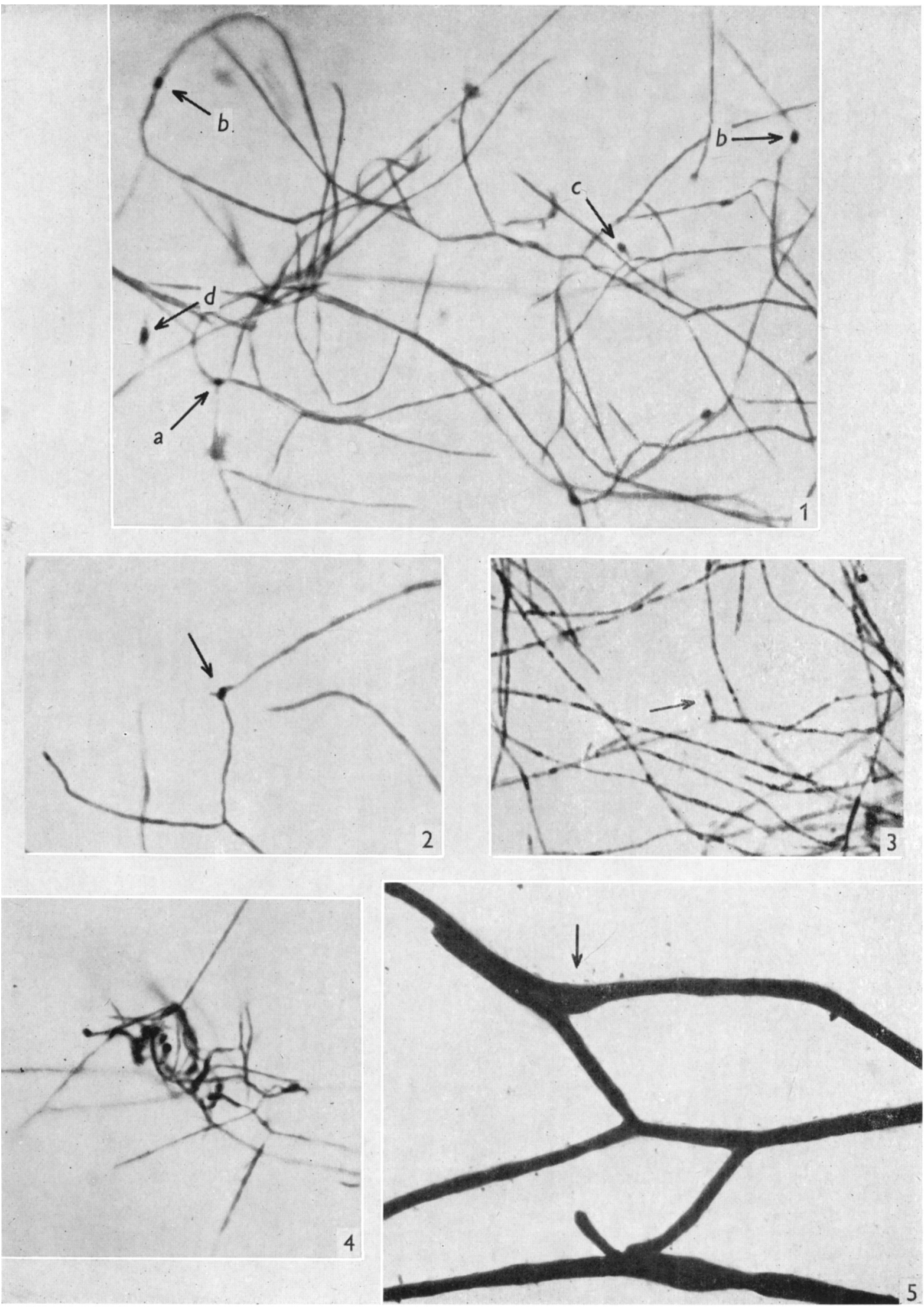

P. B. Dickenson and K. D. Macdonald-Initial clillos in streptomyces spp. Plate 1 
Journal of General Microbiology, Vol. 13, No. 1

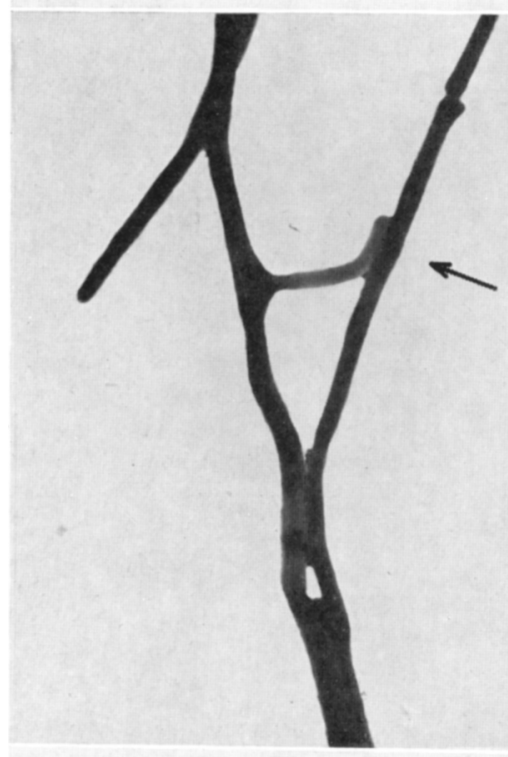

6
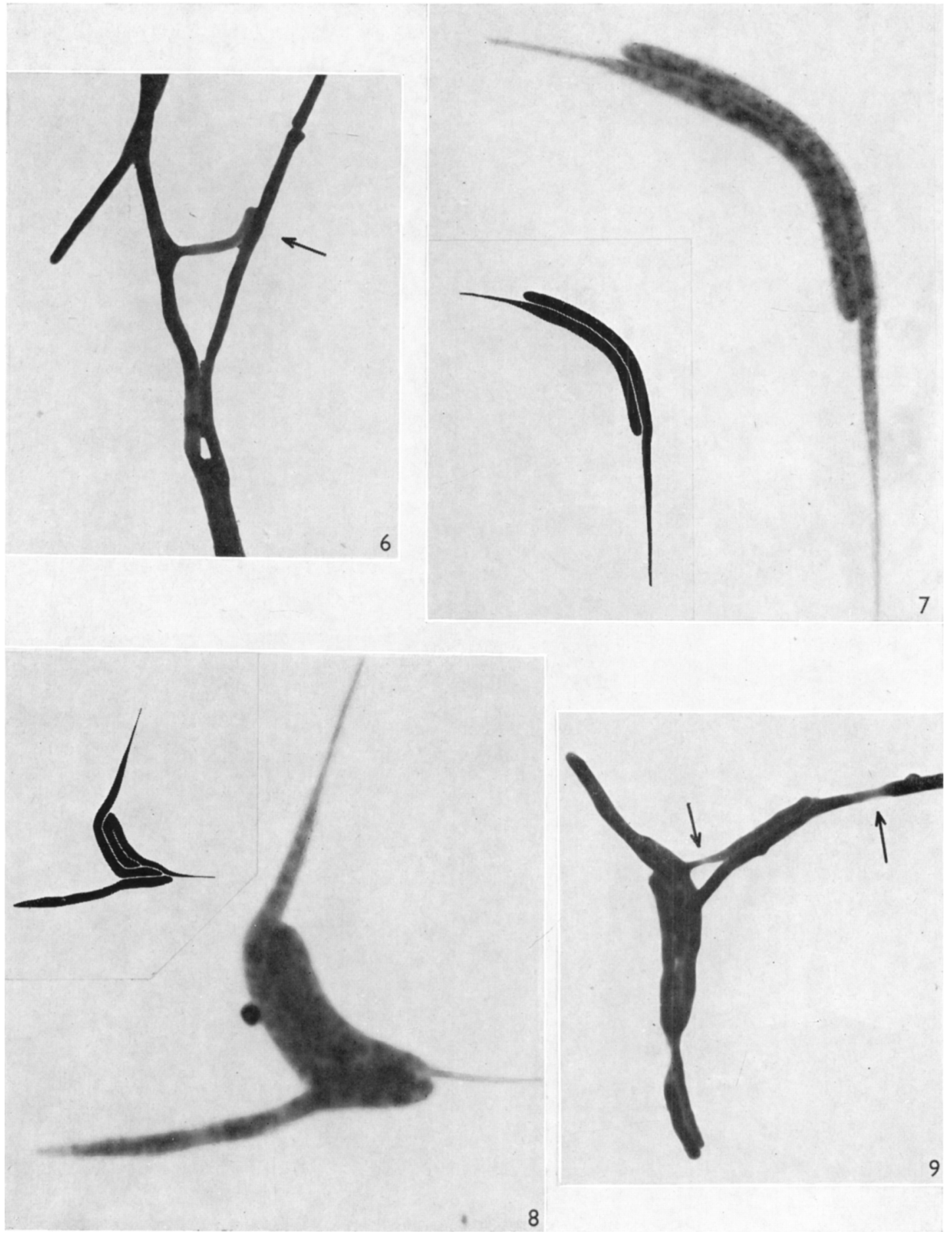

P. B. Dickenson and K. D. Macdonald-Initial cells in streptomyces spp. Plate 2 


$$
\begin{aligned}
& x< \\
& x
\end{aligned}
$$

